DOE/ER-0018
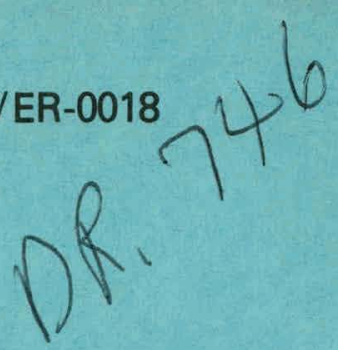

MASTER

\title{
The Department of Energy Policy for Fusion Energy
}

September 1978

U.S. Department of Energy

Directorate of Energy Research

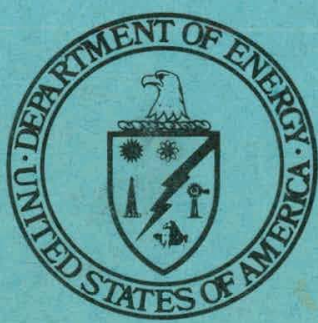




\section{DISCLAIMER}

This report was prepared as an account of work sponsored by an agency of the United States Government. Neither the United States Government nor any agency Thereof, nor any of their employees, makes any warranty, express or implied, or assumes any legal liability or responsibility for the accuracy, completeness, or usefulness of any information, apparatus, product, or process disclosed, or represents that its use would not infringe privately owned rights. Reference herein to any specific commercial product, process, or service by trade name, trademark, manufacturer, or otherwise does not necessarily constitute or imply its endorsement, recommendation, or favoring by the United States Government or any agency thereof. The views and opinions of authors expressed herein do not necessarily state or reflect those of the United States Government or any agency thereof. 


\section{DISCLAIMER}

Portions of this document may be illegible in electronic image products. Images are produced from the best available original document. 


\section{The Department of Energy Policy for Fusion Energy}

John M. Deutch Director of Energy Research

September 1978

\section{U.S. Department of Energy}

Directorate of Energy Research

Washington, D.C. 20585
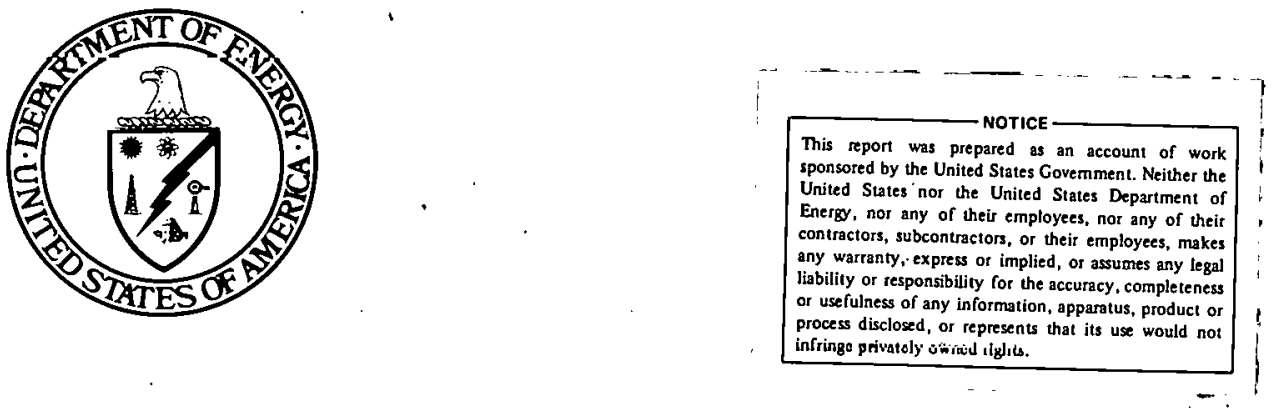

For sale Ly the Superintendent of Documents, U.S. Lovernment P’rinting Ofrice Viashington, D.C. 20102

Stock Number 061-000-00198-1 
This policy statement has been prepared under the auspices of the Fusion Review Committee of the Department of Energy's Research and Development Coordination Council

\section{FUSION REVIEW COMMITTEE}

Chairman: Dr. John M. Deutch, Director of Energy Research

Members: Mr. Robert D. Thorne, Assistant Secretary for Energy Technology.

Dr. Duane C. Sewe11, Assistant Secretary for Defense Programs 


\title{
The Department of Energy Policy for Fusion Energy
}

\author{
John M. Deutch \\ Director of Energy Research
}

The Department of Energy's applied research program in controlled thermonuclear fusion has recently been the object of public attention because of the announcement of encouraging research results. At the same time, many people working in the program have felt concern about it because of new review processes and Federal budget deliberations. Both these sources of interest make it appropriate to describe the Department's policy for fusion energy research and development, with broad emphasis on objectives and priorities.

Because the program comprises two substantially different approaches - magnetic confinement and inertial confinement of fusion reactions - we must deal with several categories of policy matters: general objectives and strategy; policies specific to each of the two programs; and finally policies governing program review.

\section{Context of the Program}

The bulk of the Department of Energy's budget.must be devoted to the solution of immediate and mid-term energy supply problems (including environmental control), primarily to the introduction of liquid fuel substitutes, 
rather than to a more distant, long-range solution. We could hardly expect it to be otherwise.

Possible long-range solutions - that is, solutions which rely on inexhaustible sources of energy - all demand technologies which do not entirely exist today or are evidently too costly. So, we cannot rely heavily on such possibilities to help us out of energy difficulties during the next 20 to 30 years. When we speak of "inexhaustibles," then, we are talking of what we: hope will be the next century's primary energy sources. While research and development programs involving inexhaustibles must compete for funds with projects of more immediate concern, it would be a mistake to infer that the Department does not view the fusion program as one of great signif jcance.

Before the middle of the next century -- sometime between 2020 and 2050 , by current estimates -- diminishing reserves of oil, natural gas, and fissile fuels will force us to place increasing reliance on inexhaustibles. But the inexhaustịbles all require many years of development before they can make the substantial contrihutinns, 
There are three such inexhaustible sources: solar energy, fission breeder reactors, and fusion. Solar energy technologies do exist now, but most wịll require long development periods before they can generate large amounts of power economically. Fission breeder technology still requires years of development and demonstration, and it still faces the political and social problems of proliferation and long-term waste disposal.

Fusion energy is furthest of the three from practical. economic utility, but its potential rewards are great. Successful commercialization of fusion could provide an energy resource whose ultimate fuel (deuterium extracted from water) is cheap and essentially unlimited, and whose by-products would pose much reduced environmental problems compared to coal and fission power. Fusion power stations would pose no increased risks to the community (beyond those of an ordinary fossil fuel plant), and would face no geographical limitations. But there is no lead time to spare: if fusion energy is to be available when it is needed -- thousands of Megawatts economically produced about 2030 -- the research and development program, rigorously directed toward the goal of commercial utility, must be undertaken in earnest now. 
In fact, the high priority which the Department places on fusion research has recently been demonstrated tangibly. We expect the total budget for fusion to remain essentially at its present level - a total for magnetic and inertial confinement of about five hundred million dollars per year -through fiscal year 1980. But the President has declared that the Federal budget will decline, and the Department of Energy will contribute a cut in spending. That the fusion program has been exempted from even a straight-percentage share of this cut is a strong affirmation of support. In the magnetic confinement program, the technology base is actually growing, because a smaller fraction of the budget is applied to the construction of large facilities.

This support has been strengthened through a 6-morth process of formal review under the Fusion Review Committee, which was established by the Department's Researoh and Development Coordination Council. That review found the program to be technically sound and to possess promise of achieving economical f'usion power eventually.

Decreases in funding from the current level could seriously endanger that promise, and leave us with an expensive research program but very little chance of developing practical fusion energy sources. 
At the same time, increases in the budget could increase our confidence in the program's success but would not be likely (under realistic budgetary constraints) to reduce materially. the time between critical choices among technical alternatives. The reasons for these limits shall be discussed later. It should now be clear that our confidence in the program's ultimate success -- its achievement of engineering and economic feasibility - is very high. But we expect that success will take many years work, more than 30 years at best. Management of the program in the interim must guarantee that it remains balanced and directed toward this product, an economic energy source.

The recent fusion review process employed two elements of import for the program's direction. First, the R\&D Coordination Council includes a standing. Fusion Review Committee composed of the Assistant Secretary for Energy Technology, the Assistant Secretary for Defense Programs and, as chairman, the Director of Energy Research. This Committee assures continuing attention to the fusion program by high-level DOE management. Second, the fusion review included a valuable contribution by the Ad Hoc Experts Group, chaired by Dr. John S. Foster. The formation of such an ad hoc group of outside experts, assembled to 
examine both magnetic and inertial fusion programs, is a clear precedent which indicates the Department of Energy's intention to benefit from expert technical evaluation from the scientific community at large during its policy formation process.

As with all other programs, the present budget represents a compromise with fiscal realities. It is important to bear in mind the very substantial research and development costs that will be incurred as the fusion program passes through the stages of technical development, from demonstration of scientific feasibility to commercialization.

Objectives

Before outlining the phases of the program, it ie necessary to be specific about its objectives.

The goal of fusion research in the Department of Energy is to develop the highest potential for employment of fusion energy.

The highest potential cannot be developed without an extensive technical base, both scientific and engineering. When choices are made, we must be confident that they are based on a firm understanding of all significant technical alternatives. This 
differs from a crash project, in which one strives to produce something that will work as soon as possible, subject only to a few minimal criteria of performance. The first device that would work at all might be far from fusion's highest potential, and the research that led to its construction would be narrowly focused.

Thus, our philosophy in designing and managing this R\&D program is to provide demonstration of that highest potential as soon as possible in an economically acceptable way that. fully preserves and develops the technical base. This cannot be accomplished in a sudden plunge ahead, giving all or most resources to the leading prospect at some particular time. We must assure that fusion technology develops toward an economical and practical engineering system in a balanced progression and provides as wide a range of choices as possible throughout the progression.

Though most people in the program should look toward fusion power - that is, a pure fusion reactor - as its goal, we must consider other applications as well. The phrase fusion energy is meant to include fusion power, and also various hybrid fusion concepts: devices in which, for example, fusion reactions are used to breed fission fuels, or to produce other useful fuels, such as hydrogen. 
Design of the Program

In the past, attention within the program has been centered on the demonstration of "scientific feasibility," which is generally accepted as attainment of net energy gain from fusion reactions. It has been felt, with justification, that until such demonstration has been made fusion energy will seëll d distant doubtful prospect. "Scientific feasibility" is necessary as the first unequivocal step in demonstrating the feasibility of controlled fusion reactions to be harnessed in a practical, economic technology.

Our confidence in making that demonstration in about 5 years is high, based on the program's steady progress over the past few years, including such gratifying results as the recent achievement of high ion temperatures in the PLT tokamak.

But we must take care not to become myopic. Demonstration of scientific feasibility is an intermediate objective, a necessary but not sufficient prerequisite for achieving the goal. As the first major objective, scientific feasibility does have the highest single priority within the program, but this priority is not granted at the expense of other objectives. Projects which aim beyond that demonstration will be supported, and in some cases expanded. 
Following the demonstration of scientific feasibility, the program will move from applied research into a development phase. Engineering Test Facilities (ETF) will be constructed for the most promising prospects in both magnetic and inertial confinement. These facilities will be integrațed systems producing net energy gain using fusion plasma techniques developed in the previous generation of experimental devices. The Engineering Test Facilities will also establish the technological requirements of each of the major components of a prototype reactor.

The next phase of the program, demonstration, will involve the operation of an Engineering Prototype Reactor (EPR), which first combines the elements tested in the superior Engineering Test Facility into a pilot plant in which the unknowns of reactor design can be tested and resolved. The EPR will approach for the first time complete energy gain, where the energy produced exceeds all energy consumed in keeping the entire plant running. Finally, demonstration will be completed with the construction of one or more commercial demonstration reactors, in which net power gain in excess if 100 Megawatts per plant is produced with an economic efficiency that will make them attractive to 
industrial investors. Full commercialization of fusion energy will have been accomplished when about one tenth of a quad per year, which is about the equivalent of three. 1,000 Megawatt power plants, is produced; we call this the Initial Operating Capability.

The Government will remain the primary source of funding through the construction and operation of the Engineering Prototype Reactor. One need not stress that the Government will wclcome inveslments by and sooperative ventures with industry and utilities as the program moves through development to demonstration.

We confidently expect that scientific feasibility will be demonstrated within the magnetic confinement program in the early to mid-80s. This will be accomplished by the Tokamak Fusion Test Reactor (TFTR), presently under construction at Princeton.

Although one cannot yet be quite as confident in preristing particular dates for sucress in inertial confinement experiments, it is possible that by 1986 scientific feasibility will have been demonstrated by one or more of 
three systems: the Nova laser at Livermore, the Antares laser at Los Alamos, and the EBFA electron beam at Sandia Laboratories. I shall not discuss here the objectives or schedule of the concurrent project to develop military applications of inertial fusion.

After scientific feasibility of its general approach has been demonstrated, each program will decide which of its competing alternatives will be the basis of its Engineering Test Facility. The two decisions will necessarily involve somewhat different logic.

In magnetic confinement, we expect that tokamaks, such as Princeton's TFTR and General Atomic's Doublet III, will have demonstrated the feasibility of operation of a tokamak ETF. The question will then be: do alternative magnetic confine-. ment schemes, particularly mirror machines, offer significant advantages over tokamaks? All such alternative devices are, so far, well behind tokamaks in achieving major objectives, and tokamaks may prove to be the best devices for fusion energy reactors. But the fact that they are ahead at this stage of research is not conclusive, and our program design must not force us to make decisions too soon. If one or 
more of the alternative schemes produces highly promising results in the interim, it is imperative to delay the decision date for the magnetic ETF in order to make the most reasonable choice - the one which will develop the highest potential for commercial fusion energy.

The situation in inertial confinement research is, in a sense, precisely the converse. The leading candidate for demonstration of scientific feasibility - the Nova glass laser facility at Livermore l.ahnratory - is not a contonder for the inertial ETF, because glass lasers cannot attain the efficiency or the repetition rate necessary for fusion reactors. The decision on what sort of ETF to build here will involve a process called driver evaluation. This is, assuming that the feasibility of inertial confinement fusion of small pellets driven by a large energy source has been demonstrated, it will then be necessary to choose the best large energy source to drive the pellets to reaction. Projects are underway to investigate various gas lasers; electron beams, and light and heavy ion beams as drivers. There is no guarantee that any one of them can be made to work as a practical fusion driver; nor can we be sure that 
when inertial scientific feasibility is demonstrated, the best candidate drivers will have demonstrated their practicability. Again, we must be prepared to suspend judgement to guarantee that we have selected the system which will.... satisfy commercial requirements.

The current plan calls for final driver evaluation to take place after 1986, and for an ETF decision to be made the following year, if the state of research permits.

We expect about 8 years to elapse in each program between the ETF decision and initial operation of the facilities. Thus reasonable dates for operation of the two ETFs are 1992-5 (for magnetic) and 1995-8 (for inertial), assuming funding follows the current projection. That projection keeps the entire program's operating budget roughly constant in 1979 dollars and estimates each ETF at about $\$ 600$ million. Between now and the time both ETFs are operational in the late 90 s, we will have spent about $\$ 11$ billion. In order to be in a position to make ETF decisions in the mid-to late-80s, and thus to have ETFs operating in the mid-90s, attention to engineering development must be intensified now. 
Experience with the ETFs will teach us, what sort of system -. magnetic or inertial confinement.-- we want to make the basis of the Engineering Power Reactor, and how to build it. That construction will take another 8 years and will cost in the neighborhood of a billion dollars. The EPR should be fully operational by 2005 , with the decision on the design and funding of the commercial demonstration reactor in progress. At that point the entire fusion program will have expended about $\$ 18$ billion.' The Initial Operating Capability would accordingly be estimated as 2020-2030, a time frame consistent with current predictions of the necessity for large contributions from alternative energy sources.

The program schedule and its milestones are shown sohematioally on the accompanying figure. This current projection of the program's timetable shnuld not be taken to be immutablé, for several reasons. First, we are sensitive to the fact that both scientific and engineering uncertainties can influence the pace and character of the program. Still, over two decades of progress in magnetic confinement research, and about half as much in inertial, justify reasonable confidence in the establishment of these planning milestones. This confidence is exists not only within the Department of 
Energy management, but in the scientific community, as the report of the Ad Hoc Experts Group shows. Second, we must recognize that this schedule can be modified by the rate at which resources are committed to the program. And third, we must be constantly prepared to accommodate substantial innovation: the program's schedule must be sufficiently flexible to include the introduction and exploration of entirely new conceptual approaches.

All these considerations point to the necessity of assessing the status and prospects of the programs as realistically as possible, and of focusing on the near term of the projected schedule as a means of maintaining flexibility and preventing premature commitment to a single approach. The important near-term decision points concern the ETF scope and the choices among ETF design prospects. At least until that point is reached, we must continue to proceed on a broad front, which means intensive investigation of many conceptual approaches. We recognize as valuable assets the program's momentum and the expertise of the corps of researchers providing it; they are assets acquired at great cost, and we shall insure their continuity both in the DOE laboratory system and in outside technical institutions. 
As was explained in discussing the program's objective, proceeding on a broad front also means that, through ETF operation and beyond, we must support a vigorous research base in plasma physics and engineering. Maintaining plasma physics research involves the familiar problem of protecting the technical base in a program which involves large and expensive hardware. Both the magnctic and inertial programs are continuing to build expensive machines, useful devices which are capable of absorbing money and talent out of supporting research at precipitous rates. We must not permit ourselves to become so preoccupied with the achievement of milestones on large devices that we have no resources devoted to understanding what is really happening, and thus to maintaining progress when something goes wrong. If we occasionally find that the research base or balance cannot be guaranteed without postponing for a year or even 2 years the operation date of a large facility, we must be prepared to face that postponement.

Increasing the engineering research base commands an equally high priority. Engineering and materials test projects, coupled w1th system designs and analyses by engineering experts, will be expanded in both programs. And as the 
programs mature from applied research to engineering development, we must encourage a shift in machine design and construction from DOE laboratories and universities to industry. Analysis and development of hybrid reactor concepts must be considered integral to an effective engineering research program.

One note of caution must be added concerning the significance of projected schedules and milestones. Fusion advocates and supporters of particular fusion schemes and approaches understandably are tempted to "oversell" the program by publicly emphasizing dramatic milestones. In a program that is both so costly and so important to our Nation, it is necessary to encourage a responsible and sober appraisal of anticipated and achieved technical progress. We must be careful also to distinguish between real scientific objectives, which involve proof of our increased understanding of systems, and the necessary technological milestones, which involve tests of high performanse of large experimental devices. The maturation of scientific. and engineering bases requires that we proceed at a measured pace through the complex development process. 
In summary, the near-term objectives of DOE fusion policy emphasize:

- Demonstration of scientific feasibility

- Establishment of sound engineering development base supporting the ETF decision

- Maintenance of a strong scientific base

- Encouragement of research in alternative concepts, and scrutiny of their positions relative to the established major projects.

\section{Magnetic Fusion}

As has been said, the magnetic confinement program has been largely dominated, both technically and financially, by tokamak research. As a result of this predominance, tokamaks have recently provided a series of gratifying and promising successes.. In increasing support of alternative concepts, it is not our object, as some might say, to penalize the leader because it is the leader.

The crux of it is that tokamaks, for all their promise, appear to possess disadvantages as reactors. Their complex geometry may lead to difficulties of operation, cooling 
and maintenance. It will be difficult to operate them in steady-state, to control impurities efficiently and to obtain direct conversion of energy in charged reaction products. Although none of these problems eliminates the possibility of an economically competitive tokamak reactor, the coincidence of them suggests that, as we try to find methods of overcoming them by improving tokamak design, we vigorously seek new schemes which, by their conceptual nature, avoid the problems altogether.

Mirror machines present one. such conceptually attractive alternative concept, though at the moment they remain scientifically behind tokamaks in demonstrated plasma confinement capacity. The question we must answer is whether this deficit is solely a result of the greater resources and attention which have been devoted to tokamaks. It may also be possible to combine the advantageous features of toroidal and mirror systems, and several concepts which attempt to do this are being studied. Many other schemes are equally far behind the mirrors themselves, yet some of these have conceptual advantages that would make them, if they worked out, highly attractive. 
Yet if we continue to support all these schemes at basicresearch level, we are guaranteeing that none of them will be actually competitive when the magnetic ETF decision is made. So it is necessary that, over the next 2 years or so, we 'choose those alternative concepts which show the most promise of providing significant advantages over tokamaks and devote available resources to them. This winnowing process is already underway. Because of our confidence in tokamaks, we are not simply asking of each project, "Will this work?" but also, "If it does, will it be much better -- from engineering and economic points of view -than a tokamak?"

We do not expect to be able to make and operate an economically satisfactory power plant unless significant progress is made on a number of critical technical issues, among which are:

1. Detailed understanding of particle and energy confinement in tokamaks, and of scaling laws for such confinement; limitations on plasma and magnetic field density.

2. Limitations on the pulse length of tokamaks operating under burn conditions;

3. Impurity control in tokamaks; 
4. First wall conditions and lifetimes in both tokamak and mirror reactors;

5. Viability of the tandem mirror concept, including maximum plasma density and energy gain achievable;

6. Detailed understanding of particle and energy confinement in tandem and field-reversed mirrors, including radial transport electron heat conduction in tandem mirrors and magnetic islands in fieldreversed geometrics.

7. Limiting powers and efficiencies of auxiliary heating techniques for both tokamaks and mirrors;

8. Safe, practical techniques of energy removal and utility at high efficiency from any magnetic confinement systems (including, in the applicable systems, direct conversion to electricity); and of course,

9. Viability of other alternate magnetic confinement systems.

\section{Inertial Confinement}

Inertial confinement research is less mature than magnetic confinement research. We are concerned that the technology base in inertial confinement is not yet as broad, in terms of number and diversity of skillful professionals employed, nor as deep, in terms of basic physics and enginejering 
issues well in hand. The difference in time and resources expended on the problems accounts for most of this disparity. It is time to give increased emphasis to broadening and deepening the program's technology base. Specifically, we hope to establish a second major target research and design capability similar to the highly expert group at Lawrence Livermore Laboratory. We also wish to emphasize the importance of basic physics experiments - studies of the detailed mechanisms of energy coupling and implosion. These experiments can for the most part be carried out on existing devices, such as the Argus and Shiva lasers at Livermore and the Helios laser at Los Alamos, and the lasers at KMS Fusion and the University of Rochester.

Regardless of the construction schedules of larger devices, we must maximize the experimental utility of existing deviues to guarantee our solid understanding of the dominant physics issues of inertial confinement. Some of these issues we take to be:

1. Detailed understanding of absorption mechanisms on pellet targets, including plasma instabilities, preheat effects, stimulated backscatter processes and transport inhibition mechanisms; 
2. Detailed understanding of the physics of ablativepusher compressions, and of ignition processes;

3. Detailed understanding of fluid-plasma instability mechanisms in targets, and thus of requirements for uniformity of illumination;

4. Optimum pulse length and actual power regime needed to satisfy the strictures of all the preceding processes;

5. Detailed understanding of effects of these processes on wavelength scaling for laser drivers, and on particular choice of particle for other drivers, as these effects prescribe limits for driver performance;

6. Understanding of the internal physics limiting the scaling and design parameters in each of the contemplated driver. systems: $\mathrm{CO}_{2}$ lasers; other gas lasers; electron beams; light and heavy ion beams.

7. Safe, practical techniques of energy removal and utility at high efficiency from any inertial confinement system.

Increased emphasis must be placed within the inertial program upon research on complete systems - drivers and targets together. For it is the complete system which must produce practical and 
efficient energy. We cannot say that certain drivers are inherently more practical or even efficient apart from the energy-generating systems of which they must be parts; certainly the same is true of target design and fabrication.

Finally, we must consider the matter of classification of inertial confinement research. Some of this research is presently classified because of the potential military applications of this technology. At present, the near-term objective of inertial fusion work is to assist our nuclear weapons programs; however, with the demonstration of inertial confinement's scientific feasibility, we would expect to see a split to an energy technology program, and a military applications technology. In the interim, we are seeking the most reasonable way to define classification priorities, a way that permits maximum participation in the research while guaranteeing the seourity of the senoltive mililary information:

Review and Coordination

The formation of the Department's Fusion Review Committee and the efforts of the Ad Hoc Experts Group demonstrate the Department of Energy's commitment to continuing review of the research and development program in controlled fusion. 
The review process has proved to be of great benefit. to the program in a number of ways. Communication and cooperation between magnetic and inertial confinement research has been improved. Perhaps most important, the program has taken a major step toward the establishment of the technical and managerial stature it deserves with other members of the scientific community, administrators and legislators.

We therefore intend to continue the Fusion Review Committee, and we shall periodically obtain advice from external experts of stature in the scientific and engineering communities. These further Ad Hoc Experts Groups may be constituted as parts of the soon-to-be established Energy Research Advisory Board. The Groups' primary responsibility will be to assist in determining the major scientific and engineering objectives of the programs and of the projects themselves, and to review regularly progress toward those objectives. The forum of the Ad Hoc Experts Group will provide an. opportunity for open review and critique of progress and plans, and will assure that individual objectives remain congruent and pertinent. 
We expect that through.such an ad hoc review process, increased cooperation between the magnetic and inertial confinement research programs will be fostered. A good interchange of plasma physics research data already exists. We intend to extend this to pooling of engineering research data and cost information for reactor studies, and eventually to the establishment of common measures of performance for proposed reactor designs and reactor subsystems.

The national laboratories will necessarily remain the centers around which the Department organizes a large part of its fusion research programs. But, as has been emphasized, integration of more scientists and engineers from the industrial and academic communities into the program, as a means of strengthening the research base, is an important and specifically designated goal. We hope also to expand our current efforts toward international cooperation in fusion research, to include more (and larger) jointly funded projects with the Japanese, Europeans, and perhaps the Soviets. Negotiations for such increased cooperative ventures are already underway. These projects would not be mere gestures of political harmony, but could be of significant value to the progress of the programs. 
We are convinced that the programs are making real progress, and that scientific feasibility will be demonstrated in about 5 years. We intend to continue development from that point to the concrete establishment of practical, economic fusion energy sources. The Department's confidence is reflected in its firm support, of the fusion budget. There is much to be done, but we can reasonably expect that fusion will become an important world energy source in the early part of the next century. 